\title{
Constrained Estimates of Multipath Covariance
}

\author{
PETER C. MIGNEREY
}

\begin{abstract}
When multipath propagation occurs, the covariance among signals traveling along rays emanating from a common source is expected to be larger than the covariance between signals generated by independent sources. Here several data adaptive constrained estimates of the covariance are derived as bilinear forms and some simulations are presented. The ability of a bilinear form to distinguish a $0 \mathrm{~dB}$ (relative to uncorrelated noise) correlated arrival pair from a $0 \mathrm{~dB}$ independent source is studied using an expected narrowband cross-spectral matrix corresponding to a simulated acoustic field with a 32 element line array at Nyquist spacing. Peaks in the covariance associated with the correlated pair of arrivals are $24 \mathrm{~dB}$ above an uncorrelated background, while no similar peaks occur for the independent source. Sidelobe ridges protruding $8 \mathrm{~dB}$ above the uncorrelated background are found to be a problem. An adaptive set of filter vectors obtained from the classical minimum variance problem are found to minimize sidelobe interference to $\mathbf{2} \mathbf{d B}$ above background, at the cost of reduced peaks having an $18 \mathrm{~dB}$ output above the uncorrelated background. A second set of adaptive filter coefficients are also obtained which minimize the power subject to the double constraint that one component of the multipath must pass undisturbed while the second component is rejected. This solution is singular along the power spectrum (diagonal) where the two constraints conflict. Off-diagonal peaks produced by the doubly constrained solution have a $30 \mathrm{~dB}$ output, while the sidelobe interference remains $2 \mathrm{~dB}$. These constrained estimates of the covariance between two arrivals of a multipath are effective in suppressing sidelobe interference. In particular the large output of the doubly constrained solution is encouraging, although a statistical anaylsis of the performance is needed.
\end{abstract}

\section{INTRODUCTION}

$S^{\circ}$ OME OCEAN ENVIRONMENTS can cause a propagating narrow-band acoustic signal to split and take several different paths from a source to a receiver. When such multipath propagation occurs, the covariance among signals traveling along rays emanating from a common source is expected to be larger than the covariance between signals generated by independent sources. Arrival angles inferred from local covariance peaks ought to be useful in determining the structure of an acoustic field.

While a physical understanding of acoustic propagation in the ocean with application to passive sonar systems was the motivation for this work, multipath propagation is a general phenomenon exhibited by any point source within some bounded field over which the wave equation is defined. Thus, other applications of this work extend to the reverberation problem in general, and in particular to electromagnetic

Manuscript received August 20, 1987; revised April 19, 1988. This work was supported by the U.S. Naval Research Laboratory, Information Technology Division as part of the Office of Naval Research Information Processing Task Area RR015-09-41, Work Unit 0387.

The author is with the U.S. Naval Research Laboratory, Washington, DC 20375-5000.

IEEE Log Number 8824099. communication and radar systems near the earth's surface. Indeed the signal processing techniques introduced in this paper are probably most useful to a radar system which is trying to sort aircraft from their ground reflections, possibly in the presence of correlated jammers.

Although several works have been published on the analysis of correlated signals, most of the attention has been directed toward eliminating the detrimental effects of correlated multipath which Cantoni and Godara [1] have shown to degrade estimates of the power spectrum. Approaches to date are mostly concerned with ways to decorrelate the multipath components. This is usually done via some variation of a spatial smoothing technique introduced by Evans, Johnson, and Sun [2] and advocated by Shan, Wax and Kailath [3], [4]. With this method, data are obtained at several locations in space and the resulting nonstationary covariance matrices are averaged together to obtain an estimate of a stationary matrix. Gabriel [5] has suggested an interesting alternative which is applicable when the various rays of the multipath have differing Doppler shifts. Under these conditions the time dependence can be exploited to achieve decorrelation.

Less attention has been directed toward estimating the covariance between two rays. The first modern step taken in this direction was by Schmidt [6] in his landmark paper on the MUSIC algorithm in which he discussed the processing of polarized sources. This work was then extended by Zoltowski and Haber [7] to include an arbitrary number of correlated rays. Unfortunately the results are disturbed by sharp "sidelobe" ridges which are induced by the presence of isolated uncorrelated sources.

In this paper, several data adaptive constrained estimates of the covariance are derived begining with an estimation of the narrow-band cross-spectral matrix. The average power spectrum, given by a quadratic form, is shown to be an estimate of variance. These basic results are then extended to include an estimate of covariance which is given by a bilinear form.

Because sidelobes of the conventional bilinear form are too large for this estimate to be useful, two alternate bilinear forms are derived which make use of constrained filter vectors. These filter vectors are obtained as solutions to two constrained minimization problems which pass one component of the multipath while minimizing or, following the suggestion of Cantoni and Godara [1], entirely eliminating the other.

Some simulation results are presented which show how each estimate discriminates a correlated arrival from the sidelobes of an uncorrelated arrival. Statistical properties along with any consequent conclusions concerning detection performance of these covariance estimates are left untreated. 


\section{Average Power Spectrum}

We begin by estimating the average power spectrum of ship noise which is presumed to be a superposition of harmonic components generated by the lightly damped vibration of ship structures, and driven by the steady-state operation of machinery. The resulting pressure fluctuations about some mean hydrostatic pressure are then reasonably assumed to be governed by a zero mean stochastically continuous process which is at least second-order stationary and therefore has a Fourier-Stieltjes spectral representation as an orthogonal process. The integrated power spectrum of this process is continuous at all frequencies, except possibly zero, because a combination of damping and acoustic loading precludes an infinite quality factor. In this model all narrow-band sources have some measurable bandwidth.

The pressure fluctuations are received by an array of hydrophones, converted into voltages, low-pass filtered, sampled and digitized as a real-number time series, $r\left(x_{j}, t_{n, m}\right)$. The samples from each hydrophone are organized into a set of $N$ sequences with $M$ samples per sequence which are obtained at equally spaced times

$$
\begin{aligned}
t_{n, m} \equiv(n M+m) \delta t, n & =\{0 \cdots N-1\}, \\
m & =\{0 \cdots M-1\}, \delta t=\text { time interval. }
\end{aligned}
$$

For the simulations presented in the following sections an expected narrow-band cross-spectral matrix is used which corresponds to a sample matrix with infinite degrees of freedom, i.e., $N \rightarrow \infty$ and $M \rightarrow \infty$. For simplicity, $J$ hydrophones are chosen to lie along a straight line at uniformly spaced positions

$x_{j} \equiv j \delta x, j=\{0 \cdots J-1\}, J=32$,

$\delta x=$ hydrophone spacing.

Each sequence beginning at time $t_{n, 0}$, is then transformed into the frequency domain using a real to complex fast Fourier transform (FFT) algorithm,

$$
r\left(x_{j}, t_{n} ; f_{q}\right) \equiv \sum_{m=0}^{M-1} r\left(x_{j}, t_{n, m}\right) \exp (-2 \pi i q m / M) .
$$

The Fourier coefficient $r\left(x_{j}, t_{n} ; f_{q}\right)$ associated with frequency $f_{q}=q \delta f, q \in\{1 \cdots M / 2\}, \delta f=1 / M \delta t$, is a zero mean second-order stationary random variable because the FFT is a linear transformation of zero mean second-order stationary data. Furthermore the basebanded time series of Fourier coefficients, $\left\{r\left(x_{j}, t_{n} ; f_{q}\right)\right\}, n=\{0 \cdots N-1\}$, fixed $q \neq 0$, fixed $j$, has a continuous integrated spectrum at all frequencies including the basebanded zero frequency. This is true because the original time series has a continuous integrated spectrum for all frequencies except possibly a zero frequency component which has been filtered out. Therefore sufficient ergodic conditions exist for this time series of Fourier coefficients to be used as an ensemble. It is convenient to drop the explicit frequency dependence and collect all Fourier coefficients obtained at one specific time into a column vector

$\mathbf{r}_{n} \equiv\left[r\left(x_{j}, t_{n} ; f_{q}\right)\right], j=\{0 \cdots J-1\}$, ensemble member $n$, implicit $f_{q}$.

Classically this vector of Fourier coefficients is linearly filtered using a discrete Fourier transform to pass one specific wavenumber associated with a particular plane wave angle of arrival $\theta$, with a steering vector given by

$\mathbf{e}\left(\theta ; f_{q}, c, \delta x\right) \equiv\left[\exp \left(2 \pi i j\left(f_{q} \delta x / c\right) \cos (\theta)\right)\right]$,

$$
j=\{0 \cdots J-1\},
$$

where $c$ is the sound speed. It is convenient to define the nondimensional wavenumber

$$
K \equiv\left(f_{q} \delta x / c\right) \cos (\theta),
$$

which allows the plane wave steering vector to be written as

$$
\mathbf{e}(K)=[\exp (2 \pi i K j)], \quad j=\{0 \cdots J-1\} .
$$

In this coordinate system for $K$, an endfire plane wave at Nyquist frequency has the nondimensional wavenumber $K=$ $\pm 1 / 2$. The filtered signal is then given by the complex vector inner-product

$$
F_{n}(K) \equiv \mathbf{e}(K)^{+} \mathbf{r}_{n},
$$

where the plus sign denotes a complex conjugate transpose row vector. Since $F_{n}(K)$ is a linear combination of Fourier coefficients, it is a zero mean second-order stationary random variable.

Usually this filtered signal is used to obtain an estimate of the average power spectrum,

$$
P_{0}(K) \equiv 1 / N \sum_{n=0}^{N-1} F_{n}(K) F_{n}(K)^{*},
$$

where the asterisk denotes the complex conjugate of a scalar. $P_{0}(K)$ may be interpreted as an estimate of the variance in $F_{n}(K)$. When the definition of $F_{n}(K)$ is substituted into $P_{0}(K)$ the average power spectrum is seen to be represented by a quadratic form,

$$
P_{0}(K)=\mathbf{e}(K)^{+} \operatorname{Re}(K),
$$

where the matrix

$$
\mathbf{R} \equiv 1 / N \sum_{n=0}^{N-1} \mathbf{r}_{n} \mathbf{r}_{n}^{+},
$$

is a maximum likelihood estimate of the cross-spectral matrix [8].

\section{Conventional Bilinear Form}

With the above concepts defined, an estimate of the covariance between signals arriving from two different directions, $\operatorname{cov}\left(F\left(K_{1}\right), F\left(K_{2}\right)\right)$, is given by

$$
B_{0}\left(K_{1}, K_{2}\right) \equiv 1 / N \sum_{n=0}^{N-1} F_{n}\left(K_{1}\right) F_{n}\left(K_{2}\right)^{*}
$$


Upon substitution for $F_{n}(K), B_{0}\left(K_{1}, K_{2}\right)$ is seen to be a bilinear form,

$$
B_{0}\left(K_{1}, K_{2}\right)=\mathbf{e}\left(K_{1}\right)^{+} \operatorname{Re}\left(K_{2}\right) .
$$

Notably, this covariance estimate approaches the variance estimate $P_{0}\left(K_{1}\right)$ as $K_{2} \rightarrow K_{1}$. Unlike the quadratic form, the bilinear form is complex, the amplitude is an estimate of the covariance, while the phase is an estimate of the expected phase difference between two arrivals. In the figures presented in this paper, the magnitude $\left|B_{0}\right|$ is graphed as a function of $K_{1}$ and $K_{2}$.

\section{SimUlations}

The relative ability of the bilinear form to distinguish a correlated arrival from both the sidelobes of an independent source and an uncorrelated background was studied using a simulated acoustic field which included three sources embedded in uncorrelated white noise. All three sources had the same $0 \mathrm{~dB}$ reference power level as the uncorrelated noise. $\mathrm{A}$ pair of statistically dependent sources with zero phase difference were located at $K=0.0$, and $K=0.25$, while an independent source was located at $K=-0.25$. In the limit of infinitely many random samples the cross-spectral matrix with these components is

$\mathbf{R}=\mathbf{I}+\mathbf{e}(-0.25) \mathbf{e}(-0.25)^{+}$

$$
+[\mathbf{e}(0.0)+\mathbf{e}(0.25)][\mathbf{e}(0.0)+\mathbf{e}(0.25)]^{+} .
$$

This specific representation of the simulated cross-spectral matrix is a sum of the identity matrix, $\mathbf{I}$, which describes the uncorrelated noise, and two dyads, one describing an independent sinusoid and the other describing a multipath sum of two sinusoids.

Fig. 1 shows an orthoscopic projection of the surface defined by $\left|B_{0}\left(K_{1}, K_{2}\right)\right|$ for the simulated field. Along the main diagonal stretching horizontally from $\left(K_{1}, K_{2}\right)=$ $(-0.5,-0.5)$ to $(0.5,0.5)$ is the average power spectrum which has peaks from each source, at $(-0.25,-0.25),(0.0$, $0.0),(0.25,0.25)$. There are also two other notable peaks located at $(0.0,0.25)$ and $(0.25,0.0)$ which are associated with the correlated pair of arrivals. The height of these two correlation peaks is $24 \mathrm{~dB}$ above the average background. No similar peaks show up at $(-0.25,0.0),(-0.25,0.25),(0.0$, $-0.25),(0.25,-0.25)$ indicating the source located at $(-0.25,-0.25)$ is independent of the other two sources. All of these features are in accord with the known attributes of the simulated field.

Unfortunately sidelobes from the sources complicate interpretation of the $\left|B_{0}\left(K_{1}, K_{2}\right)\right|$ surface. In particular each source is associated with a pair of sidelobe ridges, running parallel to each axis, which are $8 \mathrm{~dB}$ above the average background. These ridges occur when $\mathbf{e}\left(K_{1}\right)$ and $\mathbf{e}\left(K_{2}\right)$ are not orthogonal. To see this, consider an example in which $\mathbf{r}_{n}$ is a single noiseless plane wave $\mathrm{e}\left(K_{0}\right)$ arriving from the fixed direction $K_{0}$. For this noiseless field the cross-spectral matrix is a dyad,

$$
\mathbf{R}=\mathbf{e}\left(K_{0}\right) \mathbf{e}\left(K_{0}\right)^{+}
$$

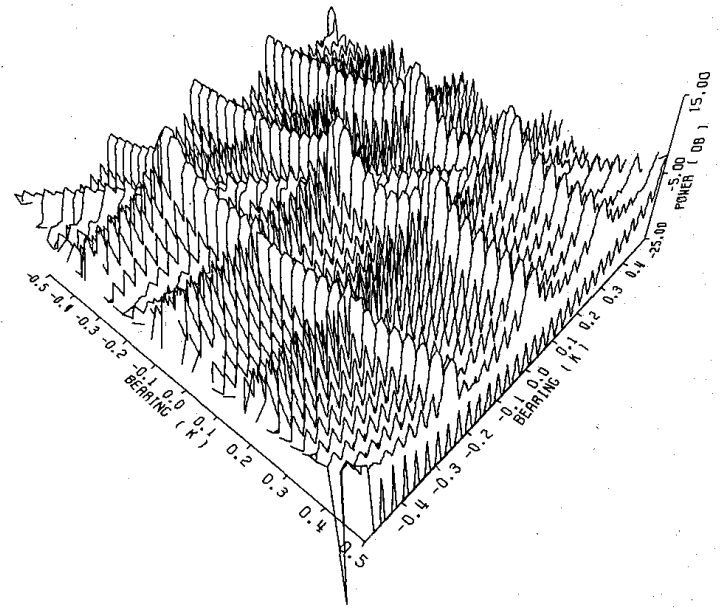

Fig. 1. An orthoscopic projection of the surface defined by the conventional bilinear form $\left|B_{0}\left(K_{1}, K_{2}\right)\right|$ as a function of the two wavenumbers being correlated. The acoustic field included a correlated multipath with components at $\left(K_{1}, K_{2}\right)=(0.0,0.0)$ and $(0.25,0.25)$ and an independent source at $(-0.25,-0.25)$. The power spectrum stretching across the horizontal diagonal from $(-0.5,-0.5)$ to $(0.5,0.5)$ shows peaks associated with the three sources. Off-diagonal peaks at $(0.0,0.25)$ and $(0.25,0.0)$ arise from the multipath. Appreciable sidelobe ridges radiate from each source along lines parallel to both axes.

which produces a bilinear form composed of two inner products,

$$
B_{0}\left(K_{1}, K_{2}\right)=\left(\mathbf{e}\left(K_{1}\right)+\mathbf{e}\left(K_{0}\right)\right)\left(\mathbf{e}\left(K_{0}\right)^{+} \mathbf{e}\left(K_{2}\right)\right) .
$$

Along a ridge where $K_{1}$ is held fixed at $K_{0}$, the magnitude of the sidelobe interference is dependent on the size of the inner product $\left(\mathbf{e}\left(K_{0}\right)^{+} \mathbf{e}\left(K_{2}\right)\right)$; likewise along the dual ridge $K_{2}=$ $K_{0}$, the sidelobes are produced by the inner product $\left(\mathbf{e}\left(K_{1}\right)^{+} \mathbf{e}\left(K_{0}\right)\right)$. Thus the magnitude of the bilinear form is generally nonzero, excepting $L$ nulls, along the sidelobe ridges produced by a single noiseless plane wave. In the following sections, an adaptive set of filter vectors are obtained which reduce sidelobe interference by minimizing the inner product $\left(\mathbf{e}\left(K_{0}\right)^{+} \mathbf{e}\left(K_{2}\right)\right)$.

\section{Minimum Variance Bilinear Form}

An obvious candidate is the classical minimum variance distortionless filter vector. This filter rejects most of the power from all wavefronts in a least squares sense, except for one specific plane wave which is allowed to pass unchanged. A constrained minimization problem may be posed for the filter vector $\mathbf{a}(K)$ as follows: find $\mathbf{a}(K)$ such that

$$
\begin{gathered}
P_{1}(K)=\min \left\{\mathbf{a}(K)^{+} \mathbf{R a}(K)\right\} ; \\
\mathbf{a}(K)^{+} \mathbf{e}(K)=1 .
\end{gathered}
$$

This problem has the well-known solution [9],

$$
\mathbf{a}(K)=\mathbf{R}^{-1} \mathbf{e}(K) /\left(\mathbf{e}(K)^{+} \mathbf{R}^{-1} \mathbf{e}(K)\right) .
$$

Because of the properties of this minimum variance solution, the two filtered signals, $F_{n}\left(K_{1}\right)=\mathbf{a}\left(K_{1}\right)^{+} \mathbf{r}_{n}$ and $F_{n}\left(K_{2}\right)=$ $\mathbf{a}\left(K_{2}\right)^{+} \mathbf{r}_{n}$, each contain a reduced amount of any one particular noise component which can inadvertently cause correlation. 


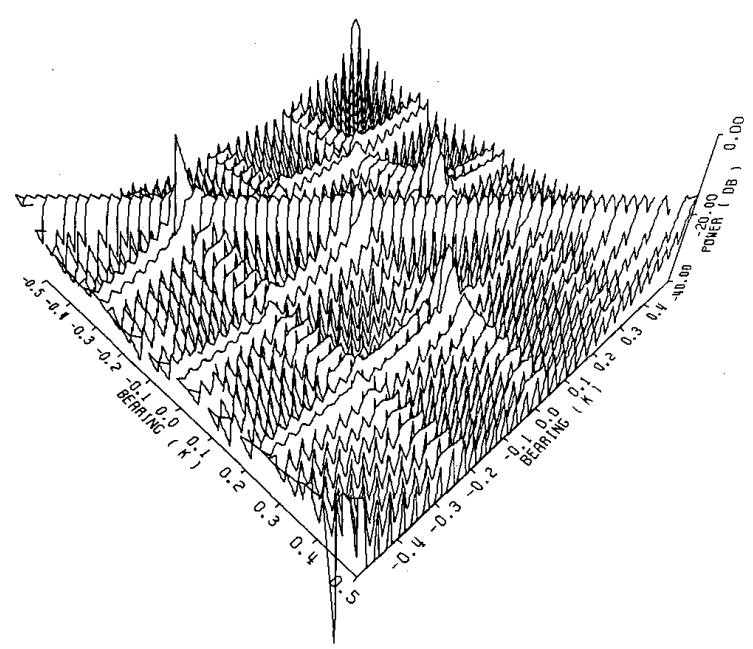

Fig. 2. An orthoscopic projection of the surface defined by the minimum variance bilinear form $\left|B_{1}\left(K_{1}, K_{2}\right)\right|$ as a function of the two wavenumbers being correlated. The acoustic field is the same as for Fig. 1. The power spectrum stretching across the horizontal diagonal has a peak associated with the independent source but fails to show the multipath peaks. Offdiagonal peaks at $(0.0,0.25)$ and $(0.25,0.0)$ arising from the multipath are degraded relative to the conventional bilinear output. Sidelobe ridges radiating from each source along lines parallel to both axes are almost reduced to the average background level.

An estimate of the covariance between these two filtered signals is given by the bilinear form

$$
\begin{aligned}
B_{1}\left(K_{1}, K_{2}\right)= & \mathbf{a}\left(K_{1}\right)^{+} \mathbf{R a}\left(K_{2}\right) \\
= & \mathbf{e}\left(K_{1}\right)^{+} \mathbf{R}^{-1} \mathbf{e}\left(K_{2}\right) /\left(\mathbf{e}\left(K_{1}\right)^{+} \mathbf{R}^{-1} \mathbf{e}\left(K_{1}\right)\right) \\
& \cdot\left(\mathbf{e}\left(K_{2}\right)^{+} \mathbf{R}^{-1} \mathbf{e}\left(K_{2}\right)\right) .
\end{aligned}
$$

Once again the solution approaches the power estimate as $K_{2} \rightarrow K_{1}=K$, only here it is the minimum variance power estimate,

$$
P_{1}(K)=1 / \mathbf{e}(K)^{+} \mathbf{R}^{-1} \mathbf{e}(K) .
$$

Fig. 2 shows the orthoscopic projection of the surface defined by the minimum variance bilinear form $\left|B_{1}\left(K_{1}, K_{2}\right)\right|$ for the same simulated field discussed above. Again the horizontal diagonal represents the power spectrum which shows a peak at $\left(K_{1}, K_{2}\right)=(-0.25,-0.25)$ corresponding to the independent source. Apparently absent from this power spectrum are any peaks associated with the two components of the multipath which one might expect to show up at $(0.0,0.0)$ and $(0.25,0.25)$. The reason for this is that the coherent sum of two plane waves is not itself a plane wave. Thus it is partially rejected in a minimum variance sense by the adaptive filter vectors [1]. Yet the filter is constrained to pass any components of the multipath which have a projection onto the steering vector $\mathbf{e}(K)$. In this simulation, the amount of multipath energy that is allowed to pass only marginally exceeds the uncorrelated noise power. The same reason explains why the two peaks away from the main-diagonal, each having a covariance $18 \mathrm{~dB}$ above the average background at $\left(K_{1}, K_{2}\right)=(0.0,0.25)$ and $(0.25,0.0)$, are degraded $6 \mathrm{~dB}$ below the corresponding output of the conventional bilinear form $\left|B_{0}(0.0,0.25)\right|$. However away from the main-diagonal, there is very little cross correlation of the uncorrelated noise to mask the reduced signal. Sidelobe ridges parallel to the axes are much reduced. Vestigial ridges which are only $2 \mathrm{~dB}$ above the average background can be noticed where nulls have been filled in.

When the single noiseless plane wave arriving from direction $K_{0}$ is reconsidered, the minimum variance bilinear form becomes

$$
B_{1}\left(K_{1}, K_{2}\right)=\left(\mathbf{a}\left(K_{1}\right)^{+} \mathbf{e}\left(K_{0}\right)\right)\left(\mathbf{e}\left(K_{0}\right)^{+} \mathbf{a}\left(K_{2}\right)\right) .
$$

Along the sidelobe ridge defined by $K_{1}=K_{0}$, the minimum variance filter, $\mathbf{a}\left(K_{1}\right)$, completely passes $\mathbf{e}\left(K_{0}\right)$ while minimizing the square magnitude of the inner product $\left(\mathbf{e}\left(K_{0}\right)^{+} \mathbf{a}\left(K_{2}\right)\right)$ as a function of $K_{2}$ in a least squares sense; again a dual relation holds along the $K_{2}=K_{0}$ ridge. So although the magnitude of the minimum variance bilinear form is minimized, it remains nonzero for a single noiseless plane wave. In the next section, an additional constraint will be applied which completely eliminates the interfering inner product.

\section{Double Constraint Bilinear Form}

Here, a set of filter coefficients are obtained which minimize the power subject to the double constraint that one component of the multipath must pass undisturbed while the second component is completely rejected. The formulation and solution of this problem closely parallels the classical minimum variance approach:

$$
\begin{aligned}
& P_{2}\left(K_{1}, K_{2}\right)=\min \left\{\mathbf{a}\left(K_{1}, K_{2}\right)+\mathbf{R a}\left(K_{1}, K_{2}\right)\right\} ; \\
& \mathbf{a}\left(K_{1}, K_{2}\right)^{+} \mathbf{e}\left(K_{1}\right)=1, \\
& \mathbf{a}\left(K_{1}, K_{2}\right)^{+} \mathbf{e}\left(K_{2}\right)=0 .
\end{aligned}
$$

In this notation the first argument of the vector function $\mathbf{a}\left(K_{1}\right.$, $K_{2}$ ) denotes the multipath component which is passed and the second argument denotes the rejected component. The resultant bilinear form is

$$
B_{2}\left(K_{1}, K_{2}\right)=\mathbf{a}\left(K_{1}, K_{2}\right)+\mathbf{R a}\left(K_{2}, K_{1}\right) .
$$

A solution is obtained by writing Lagrange's equation,

$$
\begin{aligned}
L \equiv & \left\{\mathbf{a}\left(K_{1}, K_{2}\right)+\mathbf{R a}\left(K_{1}, K_{2}\right)\right\} \\
& +\alpha\left\{\mathbf{a}\left(K_{1}, K_{2}\right)^{+} \mathbf{e}\left(K_{1}\right)-1\right\} \\
& +\beta\left\{\mathbf{a}\left(K_{1}, K_{2}\right){ }^{+} \mathbf{e}\left(K_{2}\right)\right\},
\end{aligned}
$$

which may be differentiated with respect to both the real and imaginary parts of $\mathbf{a}(K 1, K 2),(L(a)$ is nonanalytic) and set equal to zero,

$[\partial L / \partial \operatorname{Re}(\mathbf{a})+i \partial L / \partial \operatorname{Im}(\mathbf{a})] / 2$

$$
=\mathbf{R a}\left(K_{1}, K_{2}\right)+\alpha \mathbf{e}\left(K_{1}\right)+\beta \mathbf{e}\left(K_{2}\right)=0 .
$$

This equation is easily solved for $\mathbf{a}\left(K_{1}, K_{2}\right)$ when the crossspectral matrix, $\mathbf{R}$, is not singular,

$$
\mathbf{a}\left(K_{1}, K_{2}\right)=-\mathbf{R}^{-1}\left(\alpha \mathbf{e}\left(K_{1}\right)+\beta \mathbf{e}\left(K_{2}\right)\right) .
$$


The Lagrange multipliers, $\alpha$ and $\beta$, are themselves given by a system of equations which satisfy the constraints,

$$
\begin{array}{rl}
\mathbf{e}\left(K_{1}\right)+\mathbf{a}\left(K_{1}, K_{2}\right)=-\alpha \mathbf{e}\left(K_{1}\right)+\mathbf{R}^{-1} & \mathbf{e}\left(K_{1}\right) \\
- & \beta \mathbf{e}\left(K_{1}\right)^{+} \mathbf{R}^{-1} \mathbf{e}\left(K_{2}\right)=1, \\
\mathbf{e}\left(K_{2}\right)+\mathbf{a}\left(K_{1}, K_{2}\right)=-\alpha \mathbf{e}\left(K_{2}\right)^{+} \mathbf{R}^{-1} & \mathbf{e}\left(K_{1}\right) \\
- & \beta \mathbf{e}\left(K_{2}\right)^{+} \mathbf{R}^{-1} \mathbf{e}\left(K_{2}\right)=0 .
\end{array}
$$

To simplify this cumbersome notation let

$$
H_{i j} \equiv \mathbf{e}\left(K_{i}\right)^{+} \mathbf{R}^{-1} \mathbf{e}\left(K_{j}\right), \quad i, j=1,2 ;
$$

be a complex scalar such that $H_{i j}=H_{j i}^{*}$. The constraint equations may now be written in matrix form as

$$
\left(\begin{array}{ll}
H_{11} & H_{12} \\
H_{21} & H_{22}
\end{array}\right)\left(\begin{array}{l}
\alpha \\
\beta
\end{array}\right)=\left(\begin{array}{r}
-1 \\
0
\end{array}\right)
$$

After letting $|\mathbf{H}| \equiv H_{11} H_{22}-H_{12} H_{21}$, the solution for the Lagrange multipliers is

$$
\begin{gathered}
\alpha=-H_{22} /|\mathbf{H}|, \\
\beta=H_{21} /|\mathbf{H}| .
\end{gathered}
$$

This completes the solution for the constrained filter vector

$$
\mathbf{a}\left(K_{1}, K_{2}\right)=\mathbf{R}^{-1}\left(H_{22} \mathbf{e}\left(K_{1}\right)-H_{21} \mathbf{e}\left(K_{2}\right)\right) /|\mathbf{H}|,
$$

and by symmetrical argument

$$
\mathbf{a}\left(K_{2}, K_{1}\right)=\mathbf{R}^{-1}\left(H_{11} \mathbf{e}\left(K_{2}\right)-H_{12} \mathbf{e}\left(K_{1}\right)\right) /|\mathbf{H}| .
$$

When these two vectors are substituted into the bilinear form, several terms cancel to produce

$$
\begin{aligned}
B_{2}\left(K_{1}, K_{2}\right)= & -H_{12} /|\mathbf{H}| \\
= & -\left[\mathbf{e}\left(K_{1}\right)^{+} \mathbf{R}^{-1} \mathbf{e}\left(K_{2}\right)\right] \\
& /\left\{\left[\mathbf{e}\left(K_{1}\right)^{+} \mathbf{R}^{-1} \mathbf{e}\left(K_{1}\right)\right]\left[\mathbf{e}\left(K_{2}\right)^{+} \mathbf{R}^{-1} \mathbf{e}\left(K_{2}\right)\right]\right. \\
& \left.-\left[\mathbf{e}\left(K_{1}\right)^{+} \mathbf{R}^{-1} \mathbf{e}\left(K_{2}\right)\right]\left[\mathbf{e}\left(K_{2}\right)^{+} \mathbf{R}^{-1} \mathbf{e}\left(K_{1}\right)\right]\right\} .
\end{aligned}
$$

The numerator of this doubly constrained solution is the same as the numerator of the minimum variance solution discussed above. The unique properties of this solution are provided by the denominator which is zero along the diagonal, $K_{1}=K_{2}$, causing a singularity in the solution. Singularity occurs because the two constraints conflict along the diagonal; one constraint tries to pass the signal while the other tries to reject it. As a result, the doubly constrained solution can not give an estimate of the variance. However, this failure is not a major problem because any signal is known to be correlated with itself.

Simulations were conducted using the same field with three sources embedded in uncorrelated noise as discussed above. Fig. 3 shows an orthoscopic projection of the doubly constrained surface defined by $\left|B_{2}\left(K_{1}, K_{2}\right)\right|$. The power spectrum can not be computed because $B_{2}$ is singular along the diagonal $K_{1}=K_{2}$. Instead the immediate neighborhood of the power spectrum shows up as a horizontal ridge having some double peaks associated with sidelobes of the three sources.

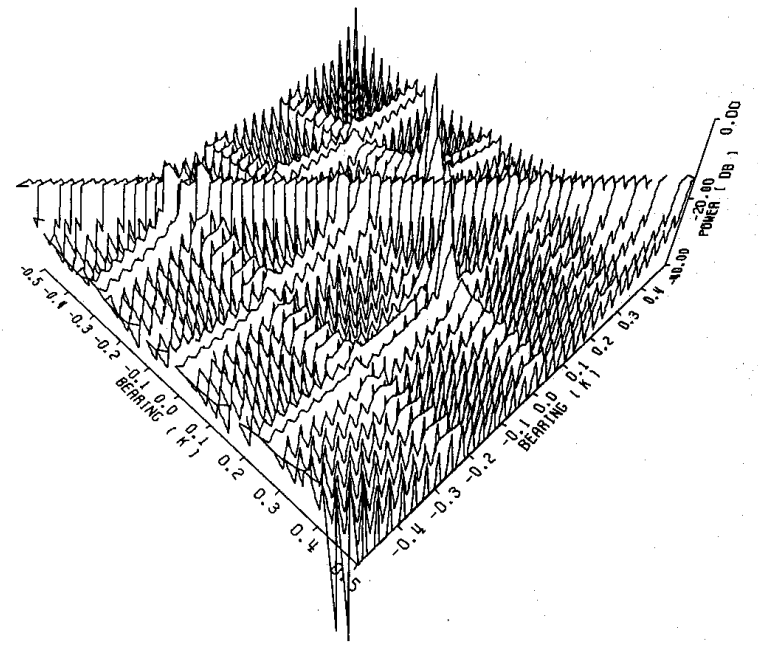

Fig. 3. An orthoscopic projection of the surface defined by the doubly constrained bilinear form $\left|B_{2}\left(K_{1}, K_{2}\right)\right|$ as a function of the two wavenumbers being correlated. The acoustic field is the same as for Fig. 1 . The power spectrum stretching across the horizontal diagonal is not shown because it is singular. Off-diagonal peaks at $(0.0,0.25)$ and $(0.25,0.0)$ arising from the multipath are larger than the Conventional bilinear output. Sidelobe ridges radiating from each source along lines parallel to both axes are the same level as those of the minimum variance bilinear form.

TABLE I

COMPARISON OF THE COVARIANCE LEVELS PRODUCED BY THE THREE BILINEAR FORMS DISCUSSED IN PAPER

\begin{tabular}{lccc}
\hline & $\left|B_{0}\right|$ & $\left|B_{1}\right|$ & $\left|B_{2}\right|$ \\
& $(\mathrm{dB})$ & $(\mathrm{dB})$ & $(\mathrm{dB})$ \\
Correlated peak & 15 & -12 & 0 \\
Sidelobe ridge & -1 & -28 & -28 \\
Uncorrelated background & -9 & -30 & -30 \\
Peak-background height & 24 & 18 & 30 \\
\hline
\end{tabular}

Two off-diagonal peaks produced by the doubly constrained solution at $\left(K_{1}, K_{2}\right)=(0.0,0.25)$ and $(0.25,0.0)$ are $30 \mathrm{~dB}$ above the average background and $12 \mathrm{~dB}$ more than the minimum variance output. Contrary to expectations arising from the second constraint, the larger output of the doubly constrained solution occurs because the peaks are taller than those of the minimum variance solution, not because the average background is lower. Indeed the average background is $-30 \mathrm{~dB}$ for both $\left|B_{1}\right|$ and $\left|B_{2}\right|$. See Table I for a summary and comparison of the various covariance levels. Included are values for the peak which indicates two correlated sources, the sidelobe ridge interference, the average uncorrelated background and the height of the peak above background. As with the minimum variance solution discussed above, the sidelobe ridges have been reduced to $2 \mathrm{~dB}$ above the average background.

\section{Vector SPACE INTERPRETATION}

It is interesting to note the similarity between the denominator of $B_{2}$ and the denominator of the estimator given by Zoltowski and Haber [7]. Both go to zero under the same conditions. To see this let $\mathbf{E}\left(K_{1}, K_{2}\right)$ be a $(J \times 2)$ direction of 


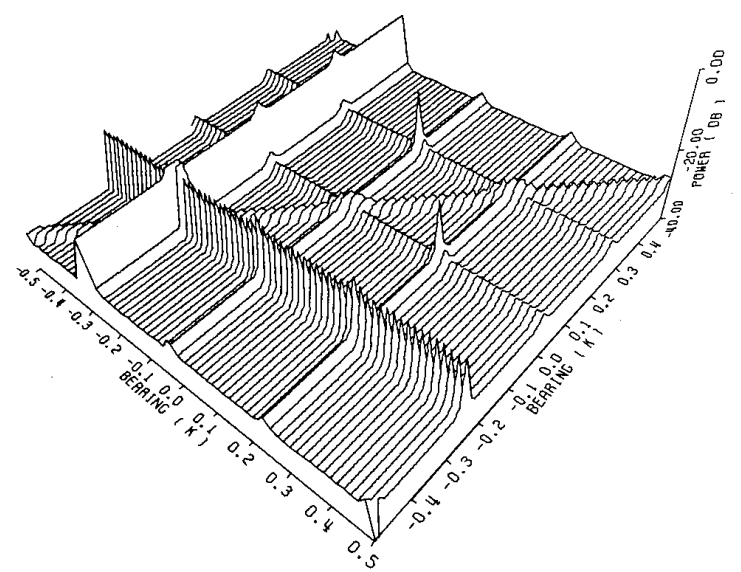

Fig. 4. An orthoscopic projection of the surface defined by $1 /|\mathbf{H}|$ as a function of the two wavenumbers being correlated. The acoustic field is the same as for Fig. 1. The power spectrum stretching across the horizontal diagonal is not shown because it is singular. Off-diagonal peaks at $(0.0$, $0.25)$ and $(0.25,0.0)$ arising from the multipath are evident. Narrow sidelobe ridges radiating from each source along lines parallel to both axes are much more prominent for a single plane wave than for the individual components of the multipath.

arrival matrix (see [7]) defined as

$$
\mathbf{E} \equiv\left[\mathbf{e}\left(K_{1}\right) \mid \mathbf{e}\left(K_{2}\right)\right] .
$$

Then the $(2 \times 2)$ matrix

$$
\mathbf{H}=\mathbf{E}^{+} \mathbf{R}^{-1} \mathbf{E},
$$

which appears in the solution for $B_{2}$, is a projection of the inverse data covariance matrix onto a subspace spanned by two potential multipath arrival vectors. A similar $(2 \times 2)$ matrix occurs in the estimator of Zoltowski and Haber with the following difference; in the above solution for $B_{2}, \mathbf{R}^{-1}$ appears in place of a MUSIC estimate of the null subspace of R. Since the determinant $|\mathbf{H}|$ is zero when the smallest eigenvalue of $\mathbf{H}$ is zero, $\boldsymbol{B}_{2}$ has poles in the same place as the Zoltowski and Haber estimator. This will occur whenever at least one source having any arbitrary multipath shape within the direction of arrival subspace is nulled out by $\mathbf{R}^{-1}$. Thus a single planewave from either direction $K_{1}$ or $K_{2}$ alone will cause the denominator to go to zero producing sharp sidelobe ridges.

Fig. 4 shows the surface defined by $1 /|\mathbf{H}|$ as a function of $K_{1}$ and $K_{2}$ which has properties very similar to the Zoltowski and Haber estimator. In particular, both of these estimators are characterized by sharp sidelobe ridges. It is then seen that the numerator of $B_{2}$ is needed to suppress these sidelobe ridges while the denominator provides the poles needed to describe the correlated arrival pair. However the numerator $\left|H_{12}\right|$ does more than just zero the sidelobe ridges because it too has off diagonal peaks in the proper location, see Fig. 5, which give $B_{1}$ the properties seen in Fig. 2.

\section{ConClusion}

Several constrained estimates of the covariance between two arrivals were derived and simulations were presented which

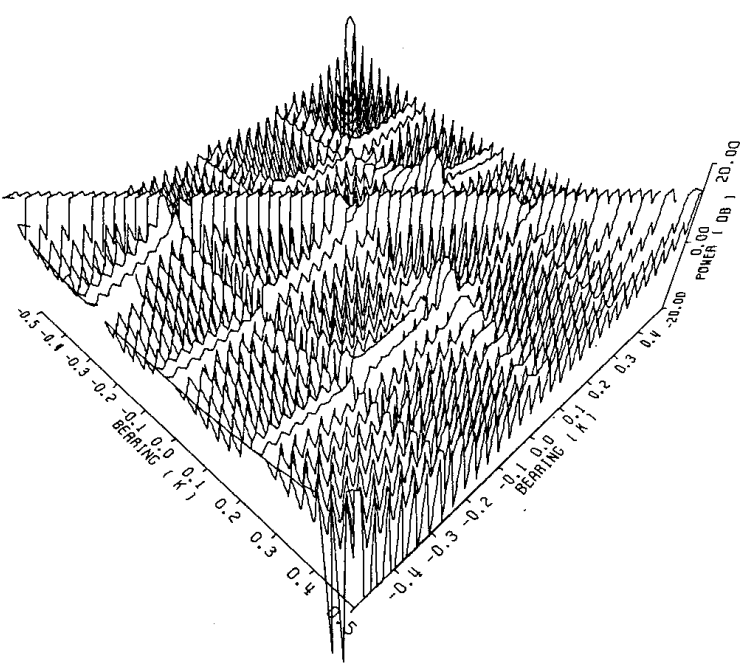

Fig. 5. An orthoscopic projection of the surface defined by $\left|H_{12}\right|$ as a function of the two wavenumbers being correlated. The acoustic field is the same as for Fig. 1. Off-diagonal peaks occur at $(0.0,0.25)$ and $(0.25,0.0)$ while nulls radiate from each source along lines parallel to both axes.

show each estimate to be effective in suppressing sidelobe interference. While the large output above the average uncorrelated background produced by the doubly constrained solution is encouraging, a thorough statistical analysis including receiver operating characteristics and Fisher matrices is needed to make a final decision on the performance of these estimates.

\section{ACKNOWLEDGMENT}

The author acknowledges several helpful discussions with Dr. Albert Gerlach and Dr. Ellen Livingston concerning various aspects of the formulation and solution of the constrained minimization problems: 


\section{REFERENCES}

[1] A. Cantoni and L. C. Godara, "Resolving the directions of sources in a correlated field incident on an array," J. Acoust. Soc. Am., vol. 67, no. 4, pp. 1247-1255, Apr. 1980.

[2] J. E. Evans, J. R. Johnson, and D. F. Sun, "Application of advanced signal processing techniques to angle of arrival estimation in ATC navigation and surveillance systems," Lincoln Lab., Massachusetts Inst. Technol., Rep. 582, June 1982.

[3] T. J. Shan, M. Wax, and T. Kailath, "On spatial smoothing for direction-of-arrival estimation of coherent signals," IEEE Trans. Acoustics, Speech Signal Processing, vol. ASSP-33, no. 4, pp. 806811, Aug. 1985

[4] T. J. Shan and T. Kailath, "Adaptive beamforming for coherent signals and interference,"' presented at IEEE Spectrum Estimation Workshop II, Nov. 10-11, 1983, Tampa, FI, pp. 55-59.

[5] W. F. Gabriel, "Spectral analysis and adaptive array superresolution techniques,'” Proc. IEEE, vol. 68, no. 6, pp. 654-666, June 1980.

[6] R. O. Schmidt, "Multiple emitter location and signal parameter estimation," in Proc. Rome Air Develop. Center Spectral Estimation Workshop, RADC-TR-79-63, Oct. 1979, pp. 243-258, Rome, NY. (Also IEEE Trans. Antennas Propagat., vol. AP-34, no. 3, pp. 276-280, Mar. 1986.)

[7] M. Zoltowski and F. Haber, "A vector space approach to direction finding in a coherent multipath environment," IEEE Trans. Antennas Propagat., vol. AP-34, no. 9, pp. 1069-1079, Sept. 1986.
[8] T. W. Anderson, An Introduction to Multivariate Statistical Analysis. New York: Wiley, 1968, ch. 3.2, pp. 44-51.

[9] R. T. Lacoss, "Data adaptive spectral analysis methods," Geophys., vol. 36, pp. 661-675, Aug. 1971 .

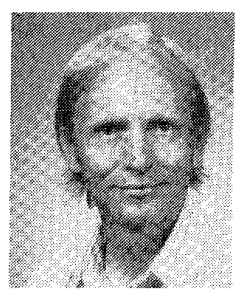

Peter C. Mignerey received the B.S., M.S. and Ph.D. degrees from the University of Rochester, Rochester, NY, in 1970, 1971, and 1979.

In 1980 and 1981 he taught and did research on ocean eddies at the University of Maryland. In 1982 he joined the Naval Research Laboratory, Washing ton, DC, as a Research Physicist assigned to the Acoustics Division. Since his arrival at NRL, he has conducted research in high-resolution spectral estimation using various techniques based on multivariate statistics including: maximum likelihood constrained minimum variance, eigenvalues and eigenvectors, and maximum entropy. He is currently concerned with the application of high-resolution signal processing methods to matched-field processing. Such processing exploits the correlated multipath structure of an acoustic field. In pursuit of this problem, he is applying numerical integration techniques to the solution of the acoustic propagation problem on a parallel computer and is developing parallel algorithms for high-resolution signal processing. 